\title{
Full circuit calculation for electromagnetic pulse transmission in a high current facility
}

\author{
Zou Wenkang, ' Guo Fan, Chen Lin, Song Shengyi, Wang Meng, Xie Weiping, and Deng Jianjun \\ Key Laboratory of Pulsed Power, Institute of Fluid Physics, China Academy of Engineering Physics, \\ P.O. Box 919-108, Mianyang 621999, China
}

(Received 4 March 2014; published 19 November 2014)

\begin{abstract}
We describe herein for the first time a full circuit model for electromagnetic pulse transmission in the Primary Test Stand (PTS) - the first TW class pulsed power driver in China. The PTS is designed to generate 8-10 MA current into a $z$-pinch load in nearly $90 \mathrm{~ns}$ rise time for inertial confinement fusion and other high energy density physics research. The PTS facility has four conical magnetic insulation transmission lines, in which electron current loss exists during the establishment of magnetic insulation. At the same time, equivalent resistance of switches and equivalent inductance of pinch changes with time. However, none of these models are included in a commercially developed circuit code so far. Therefore, in order to characterize the electromagnetic transmission process in the PTS, a full circuit model, in which switch resistance, magnetic insulation transmission line current loss and a time-dependent load can be taken into account, was developed. Circuit topology and an equivalent circuit model of the facility were introduced. Pulse transmission calculation of shot 0057 was demonstrated with the corresponding code FAST (full-circuit analysis and simulation tool) by setting controllable parameters the same as in the experiment. Preliminary full circuit simulation results for electromagnetic pulse transmission to the load are presented. Although divergences exist between calculated and experimentally obtained waveforms before the vacuum section, consistency with load current is satisfactory, especially at the rising edge.
\end{abstract}

DOI: 10.1103/PhysRevSTAB.17.110401

PACS numbers: 52.35.Mw, 52.58.Lq, 84.70.+p, 84.60.Ve

\section{INTRODUCTION}

Since the breakthrough in $\mathrm{x}$-ray power generation achieved in the 1990s when a series of wire array experiments were conducted on the Saturn generator and the $Z$-machine of the Sandia National Laboratories [1-3] that demonstrated extremely intense x-ray emission, use of $z$-pinches has been regarded as one promising route for achieving inertial confinement fusion [4-10]. In these fast $z$-pinch implosions, where several to more than $20 \mathrm{MA}$ current rises in about $100 \mathrm{~ns}$ or less, stored electrical energy can be efficiently converted into $\mathrm{x}$ rays in a pulsed-power accelerator. In such pulsed power accelerators, energy is compressed temporally with switches and pulse forming lines, and spatially by utilizing conical magnetic insulation transmission lines (MITLs) to transfer to a load [11-13]. To describe how the pulsed power components behave and what the working voltage or current are in these processes, circuit simulation and analysis are very essential. Actually, the first step in designing any large pulsed power system is to define the performance requirements and to begin an iterative design with circuit tools $[14,15]$. Then

\footnotetext{
*wenkangzou@gmail.com

Published by the American Physical Society under the terms of the Creative Commons Attribution 3.0 License. Further distribution of this work must maintain attribution to the author $(s)$ and the published article's title, journal citation, and DOI.
}

electromagnetic field simulations or particle-in-cell (PIC) simulation with the derived voltage and current level are usually followed. The two design phases are critical. Engineering designs could not start until reasonable results have been achieved in these phases.

Circuit codes are also very important during the operation phase of pulsed power systems. They can be used to understand how the components behave, and to predict the output pulses before experiments. This gives basic input for physicists to evaluate the physical results. In the past decades, with the improvements in computer capabilities, significant progress had been made in computational techniques for pulsed power design. Nowadays, multiphysical software which includes circuit, field and PIC packages is becoming commercial available [16,17] Nonetheless, full circuit simulation is still a cheap and fast way to determine the baseline for a totally new or modified pulsed power accelerator [18,19]. Circuit codes such as Bertha [20], TLCODE [21], and commercial codes such as Pspice are widely used in pulsed power design. However, sometimes time-dependent characteristics are important for some circuit element, such as switch resistance, shunt resistance in transmission lines and load inductance. Therefore, open codes which allow adding self-defined models are much favored $[22,23]$.

The $Z$ [24], the Primary Test Stand (PTS) [25-28] and the Angara-5-1 [29] research groups all have used exclusive codes to investigate accelerator power flow by introducing 
theoretical concepts for magnetic insulation or magnetohydrodynamic (MHD) models for the load [30]. In those codes, an incident wave or equivalent voltage source at the boundaries of the MITL is often used to drive an equivalent circuit thereafter. The limitation of this treatment is that, when the charging voltage in Marx generators or timing in pulse forming section changes, a new incident wave or equivalent voltage source should be used. On the other hand, in pulse shaping experiments, some switches may be closed 100-300 ns earlier with respect to the others [31]. The triggering interval of two laser switches might be larger than the electrical length between them. Then electromagnetic pulses from an early-closed module will be reflected seriously from upstream when arriving at the switches of later-closed modules. Therefore a reflection will be added to the original incident wave, and the driving waveform is totally different from the ideal synchronized-switch case.

There are some important concerns in pulse shaping experiments for pulsed power accelerators. The first one is if the operating parameters for all circuit elements are in a reasonable range. Second, to what extent does asynchrony influence power flow and azimuthal uniformity in the conical MITLs? These lead to the third concern, what is the reliable and reasonable timing span to ensure the normal function of the accelerator in this kind of experiments. Therefore, a full circuit model which includes all functional parts and takes the magnetic insulation process into consideration is necessary and useful. The merit of such a circuit model is that the driving voltage for load will be more accurate, and with a specific magnetic insulation scenario, one may know the exact time history of current loss for each MITL segment.

In this paper, a full circuit model for the PTS facility is introduced. This model allows adding time-dependent parameters for switches, MITL and load. The primary simulation results for shot 0057 and its comparison with experimental results are included as well. This paper is organized as follows. Section II gives an overview introduction on the PTS facility and its circuit model. In Sec. III, the method of pulse transmission calculation in the MITL is presented in detail. In Sec. IV, direct comparisons between the measured signals from PTS shot 0057 and numerical model results are given. Conclusions and possible refinements to the model are given in Sec. V.

\section{THE PTS FACILITY AND ITS FULL CIRCUIT MODEL}

The PTS facility is a multiterawatt (TW) pulsed power accelerator with current of 8-10 MA and rise time of about 90 ns $(10 \%-90 \%)$. The facility consists of 24 identical modules [26] electrically in parallel, which are piled up in two layers. The layout of the facility is shown in Fig. 1. A cutaway view of two modules is illustrated in Fig. 2. The two modules share an oil tank. Each module includes a Marx generator, an intermediate store (IS), a laser triggered gas switch (LTGS), a pulse forming line (PFL), a water

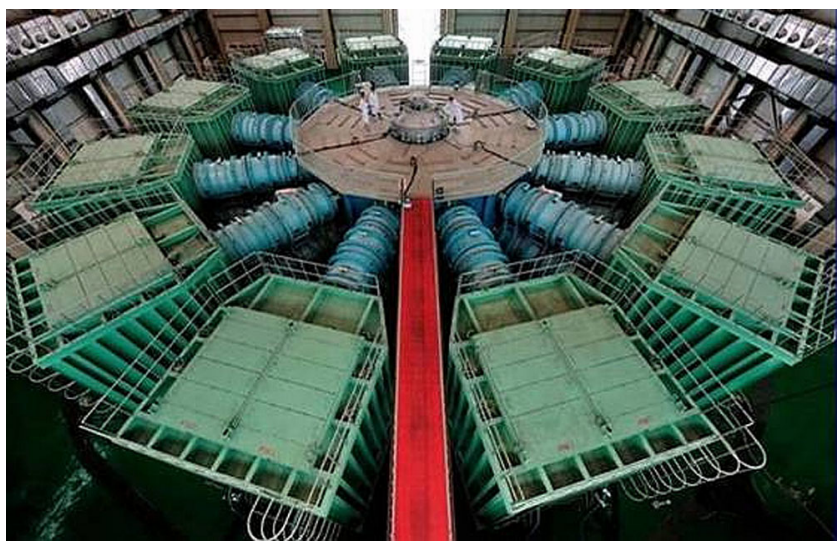

FIG. 1. Layout of the PTS facility. Twelve oil tanks (the outer most in light green) contain twenty-four Marx generators. The coaxial sections (middle in light blue) include twenty-four sets of intermediate storage, laser triggered gas switches and pulse forming lines. They are in two layers, twelve modules in each layer. In the center is the water tank and vacuum chamber. The red strip between the tanks is an aisle.

switch and a triplate transmission line (TL). The PTS uses a water tank smaller than that of the $Z$ machine. Only the 24 triplate transmission lines are placed inside. This reduces the quantity of deionized water and makes maintaining easier. After converging in the vertical triplate line, the power from the module pair is added with power from the other 22 modules and transferred downstream via a fourlevel insulation stack and a four-level conical MITL system. At the radius of about $15 \mathrm{~cm}$, current from four MITLs is summed with a traditionally used double post-hole convolute (DPHC) and then transferred to load by a short MITL.

The 24 laser triggered multigap multichannel gas switches in the PTS work at a 4 MV level and are triggered with twelve identical Nd:YAG lasers at its fourth harmonic $(\lambda=266 \mathrm{~nm})$. The maximum output energy of the laser is higher than $100 \mathrm{~mJ}$. Each laser is divided into two beams and used to trigger two adjacent switches in the same layer $[27,28]$. The jitter of the LTGS is less than $1.5 \mathrm{~ns}$. This enables stringent synchronization and precise timing for the 12 laser triggered switches. This capability of the PTS is very beneficial for pulse shaping and equation of state (EOS) experiments where specific current waveforms are needed.

In order to facilitate $x$-ray and visible light diagnoses, the target position in the PTS is located in a position higher than the working platform. This means the lower module has to use a longer transmission line than the upper one. The difference is about $30 \mathrm{~ns}$ in the vertical triplate water transmission line. Besides, in the conical MITL, the lowest level has the longest transit time and the largest angle with respect to the horizon. This leads to a difference in total inductance and voltage for the four MITLs. The difference in voltage between levels has been taken into account in designing the insulator stack.

At the beginning of the PTS design process, a full circuit simulation was conducted with Pspice, in which many 


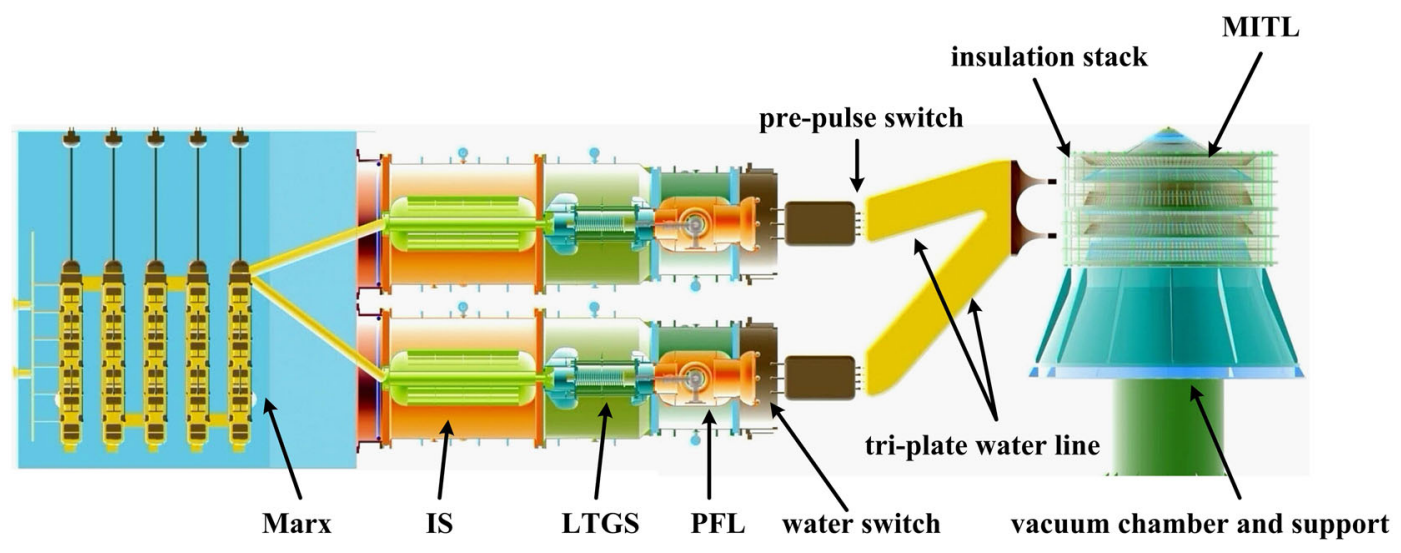

FIG. 2. Cutaway view of two modules in the PTS. The two Marx generators overlap with each other in this viewpoint. They are connected to the upper and lower module separately. The target position is higher than the insulator stack and transmission lines in the PTS to facilitate diagnostic arrangement. Thus, there is a difference of $30 \mathrm{~ns}$ between the vertical triplate water lines of the two modules.

empirical models and parameters were used. Then the basic performance requirements for each component were derived. When all key technologies for a single module were successfully demonstrated separately, a prototype module of the PTS was constructed and tested. By comparing simulation results with test data, those empirical models and parameters which were inaccurate or unreasonable in initial circuit were revised, and a new full-circuit model for the PTS was built. However, the prototype module does not have an insulation stack and MITL, so the new circuit model still had many uncertainties. Moreover, the current loss in the MITL changes with time and is parametrically dependent, which is almost impossible to model with commercial software. A new circuit model in free code is suitable for the work. With this motivation, we built a new circuit model for the PTS.

Figure 3 shows the full circuit topology for the PTS. For simplicity, each functional section in Fig. 3 is represented by a few transmission line elements. Actually, each TL element in Fig. 3 represents a subcircuit which might consist of capacitance, inductance or time-dependent resistance, or all of them. In this model, all capacitors and inductors in the Marx generators are transformed to lossless transmission lines, each with one time step $(0.1 \mathrm{~ns})$ in length. The PFLs and TLs are divided into transmission line elements with different electrical length. How many elements each part is divided into mainly depends on the uniformity in its impedance profile and the requirement in calculation accuracy. If the impedance changes fast, more elements are needed. This ensures the model is closer to reality, and better calculation accuracy may be derived. But more time and memory are needed for the calculation.

The IS and PFL both have eight transmission line elements in a module with total length of about $67 \mathrm{~ns}$ and $39 \mathrm{~ns}$, respectively. The water convolute, in which the line structure gradually changes from vertical triplate to horizontal triplate, represents a much more complex subcircuit with 25 elements. On the other hand, the MITLs have about 200 elements in all, but the total electrical length is only about $20 \mathrm{~ns}$. The purpose of using small electrical

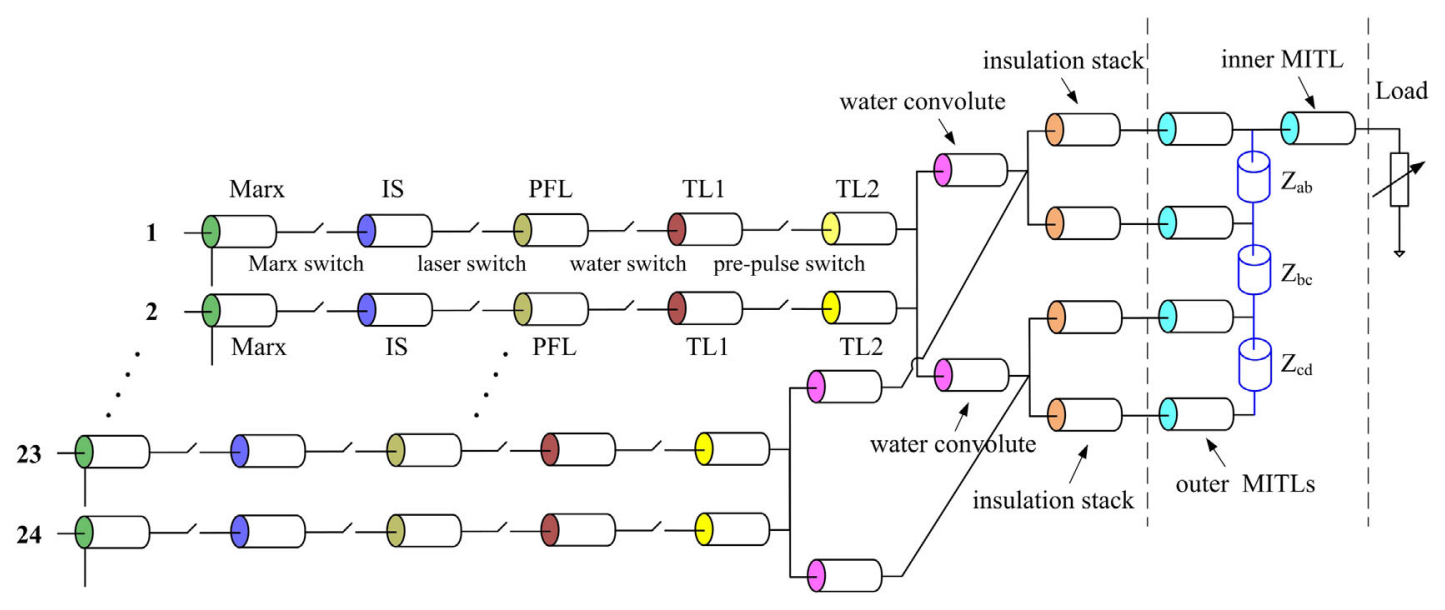

FIG. 3. Schematic model of the PTS with all twenty-four modules. All capacitors and inductors are transformed to TL elements. Each TL here actually represents a subcircuit consisting of capacitance, inductance or time-dependent resistance, or all of them. 


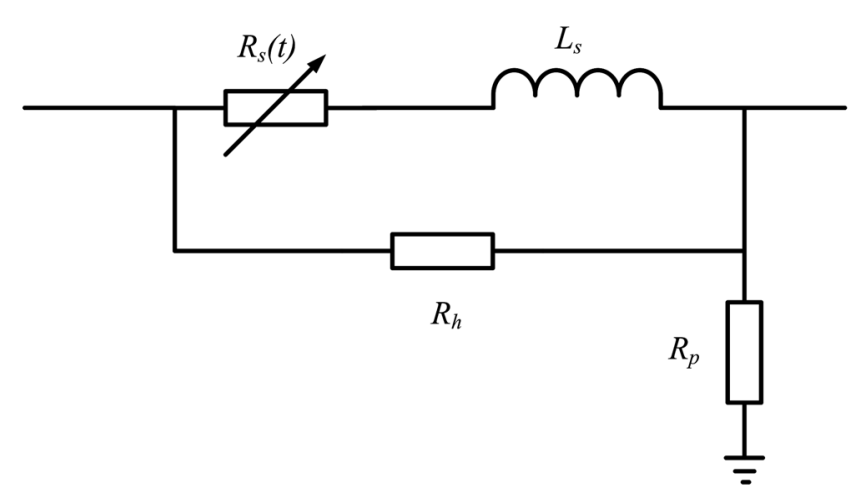

FIG. 4. Subcircuit for the PTS LTGS before transforming to a TL model. $L_{s}$ is the equivalent inductance of the switch. $R_{h}$ represents the resistance resulting from its field grading structure. $R_{p}$ is a shunt resistor account for loss.

length in the MITL is to obtain higher accuracy for current loss in space during the formation of magnetic insulation. The difference is, in the IS, PFL and TL, only a constant shunting resistance is used for all elements. But in the outer MITLs, each element has a shunting current to ground, which varies with time to account for loss. (Loss in the inner MITL can be taken into account by introducing a shunt resistance called $Z_{\text {flow }}$ as in the $Z$ machine [32]. But we have not used this model in the PTS simulation at present.)

Parasitic capacitance and inductance are also transformed to lossless transmission line elements too. But it is very difficult to determine their exact values. The major technique to derive these values is with static field calculation. Then a trial and error method is used by comparing simulations and experimentally measured waveforms from the PTS prototype module. As an example, the complete subcircuit for the LTGS in the PTS is shown in Fig. 4. In this subcircuit, $R_{s}(t)$ is a time-dependent spark resistance, which starts to change at a specific time corresponding to the arrival of the laser pulse. $L_{s}$ is the equivalent inductance of the switch. $R_{h}$ represents the resistance resulting from its field grading structure. $R_{p}$ is a shunting resistor account for loss. All these parameters were benchmarked with experimental results.

A time-dependent load model can be introduced that depends on the physical problem concerned. For example, as will be introduced in Sec. III, a 0-dimentional (OD) model for load inductance can be used to estimate the load current in z-pinch experiments. For better reliability, an MHD model for pinch resistance is favored [30].

\section{CALCULATION OF PULSE TRANSMISSION IN THE MITL}

All capacitances and inductances are transformed to TL elements, and suitable loss mechanisms are adopted for each functional part. The solution to the whole circuit network is transformed to a telegrapher-equation calculations. Then the complete pulse transmission calculation in the PTS pulsed power accelerator is possible with sophisticated TL methods [21,22]. As described above, resistors that denote MITL loss current before establishment of magnetic insulation are not constant. Therefore, the major mission for the PTS full-circuit analysis and simulation tool (FAST) code is to define the shunting current for each MITL element in every time step.

Figure 5 shows the TL model for the PTS MITL and $z$-pinch load. The outer MITLs were divided into 195 elements, each with a time-dependent resistance to indicate current loss to the anode. The four layers of the outer MITLs are linked together by the DPHC, which is indicated by three coupling inductors in the equivalent circuit model. These inductors are illustrated by TL elements 196 to 198 in Fig. 5. The inner MITL is shown as TL element 199.

The $z$-pinch load is represented with a variable inductance (TL 200) and a constant pinch resistance $R_{\text {pinch }}$. The load inductance is described with a OD model. We have

$$
L_{\mathrm{load}}=2 l \cdot \ln \frac{r_{i}}{r(t)}
$$

where $L_{\text {load }}$ is the load inductance, $l$ is the length of wire array, $r_{i}$ and $r(t)$ are initial array radius and time-dependent radius respectively. When derived, $L_{\text {load }}$ is changed to TL element 200. We use the same pinch resistance per unit pinch length as Ref. [11], which is estimated by the plasma-resistivity model developed in Ref. [33].

The basis for calculation of the electromagnetic pulse transmission in the MITL is the telegraph equation:

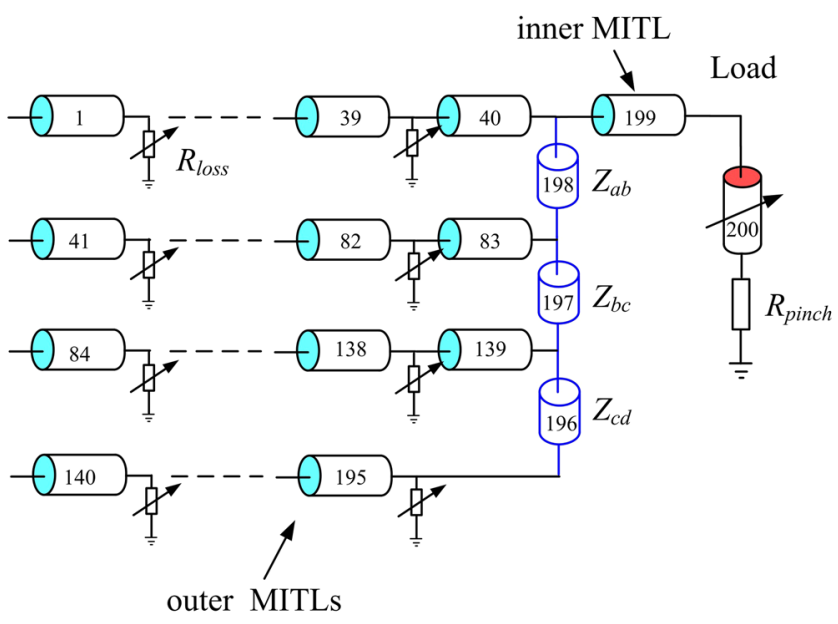

FIG. 5. TL model for the PTS MITL and $z$-pinch load. The TL elements 1 to 195 represent the outer MITLs. There is a variable shunting resistor $R_{\text {loss }}$ for each element accounting for loss current. Elements 196 to 198 are coupling inductances between different MITL levels. Element 199 is the inner MITL. All the TL elements have an electrical length of one time step (0.1 ns).The $z$-pinch load is denoted by a variable inductance and a constant resistor. 


$$
\begin{aligned}
L \frac{\partial I}{\partial t}+\frac{\partial V}{\partial x} & =0, \\
C \frac{\partial V}{\partial t}+\frac{\partial I}{\partial x} & =-J_{e}(V, I),
\end{aligned}
$$

where $L$ and $C$ are inductance and capacitance per unit length, $V$ and $I$ are line voltage and current, $x$ is the coordinate in the transmission direction, $t$ is time and $J_{e}(V, I)$ is the density of loss current to anode. A practical model for $J_{e}(V, I)$ is expressed as [34]

$$
J_{e}(V, I)=j_{C L}(V) F_{e}(Y) K(t,|E|),
$$

where $j_{C L}(V)$ is the current density given by the ChildLangmuir law [35],

$$
j_{C L}(V)=\frac{4}{9} \varepsilon_{0}\left(\frac{2 e}{m_{e}}\right)^{1 / 2} \frac{V^{3 / 2}}{d^{2}},
$$

where $\varepsilon_{0}$ is the permittivity in vacuum, $e$ and $m_{0}$ are charge and rest mass of an electron and $d$ is the gap between anode and cathode electrode. $F_{e}(Y)$ is an artificial coefficient which reflects the threshold dependence on the current. It should be considered as a qualitative function:

$$
F_{e}(Y)=\left\{\begin{array}{l}
\sqrt{1-Y^{2}}, Y<1 \\
0, Y \geq 1
\end{array}\right.
$$

and

$$
Y=\frac{I}{I_{m}(V)},
$$

where $I_{m}(V)$ is the critical current for self-magnetic insulation. $K(t,|E|)$ in Eq. (3) is a function that denotes the emission ability and depends on the electric field $E(t)$ :

$$
K(t,|E|)=\left\{\begin{array}{l}
0, E_{\max } \leq E_{1} \\
\left|\sin \left(\frac{\pi}{2} \frac{E_{\max }-E_{1}}{E_{2}-E_{1}}\right)\right|, E_{1} \leq E_{\max } \leq E_{2}, \\
1, E_{2} \leq E_{\max }
\end{array}\right.
$$

where $E_{\max }$ is the maximum cathode electrical field strength within the time interval $[0, t], E_{1}$ and $E_{2}$ are the lower and upper limit of cathode electrical field strength in which the emission ability increases from 0 to 1 .

Figure 6 shows the discretization of the MITL in the transmission direction and the equivalent TL model. For each TL element, the electrical length is 0.1 ns (i.e., one time step, corresponding to $3 \mathrm{~cm}$ in the $x$ direction), and a set of four voltage vectors are defined, each end with two. The vectors with subscript 1 and 2 denote the incoming and outgoing voltage wave respectively. For element $k$, the two ends are specified as superscript $2 k-1$ and $2 k$. We define the $k$ th TL element voltage and current at time step $n$ as

$$
\begin{aligned}
V(k, n) & =V_{1}^{2 k}(n)+V_{2}^{2 k}(n) \\
& =V_{1}^{2(k+1)-1}(n)+V_{2}^{2(k+1)-1}(n),
\end{aligned}
$$

and

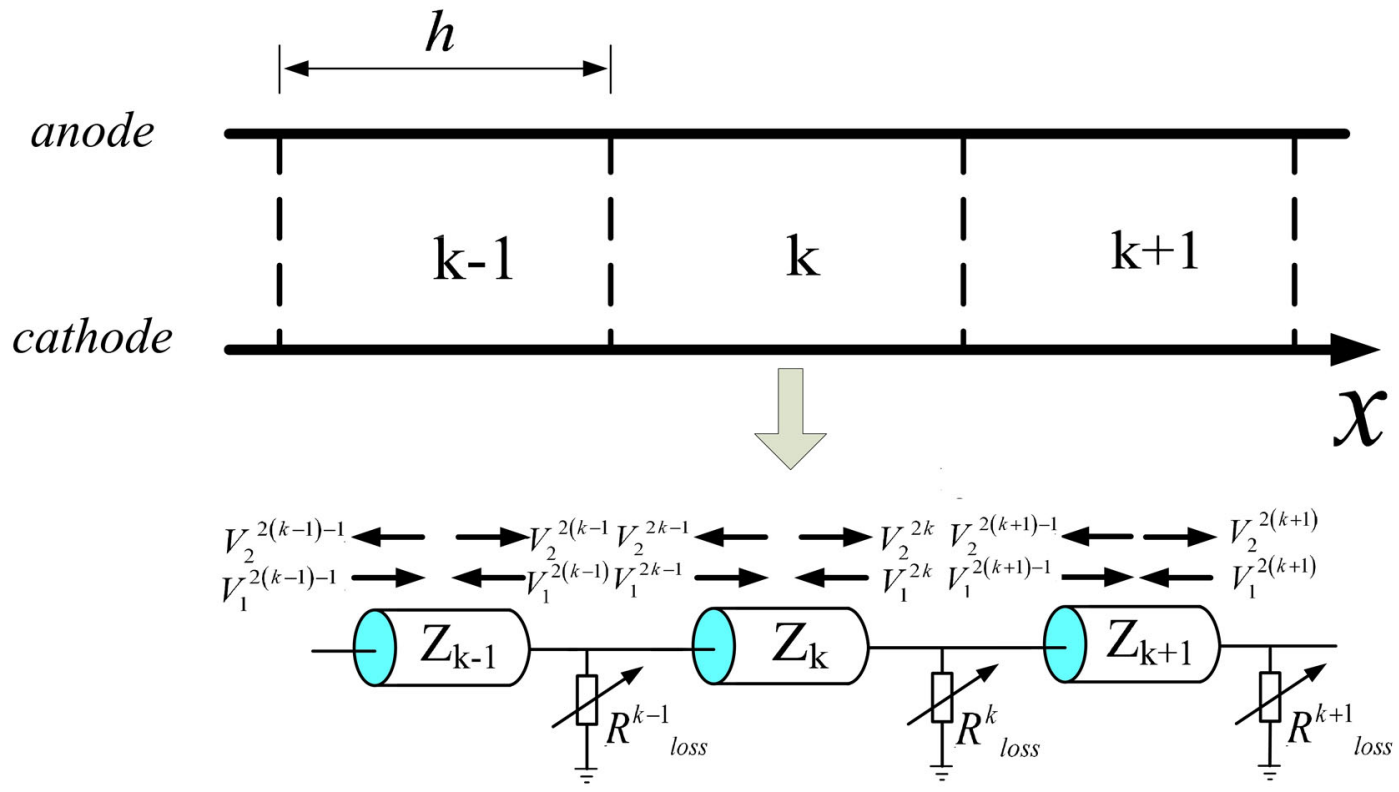

FIG. 6. Schematic of MITL discretization along the transmission direction and the corresponding TL model in the calculation. The grid size is $3 \mathrm{~cm}$ and the equivalent electrical length is $0.1 \mathrm{~ns}$ (one time step). Each TL element has four voltage vectors, each end with two. A shunt resistor is defined to account for loss current for each TL element and can be updated at every time step. 


$$
I(k, n)=\frac{V_{2}^{2 k}(n)-V_{1}^{2 k}(n)}{Z_{k}(n)} .
$$

Knowing the voltage vectors at time $n$, voltage vectors at time $n+1$ are updated with the aid of delay relations, and reflection and incident coefficients at each junction. Then the electric field on the cathode surface of each MITL element is calculated. If the electric field exceeds the explosive electron emission threshold, the MITL begins to emit electron. Otherwise, no electrons are emitted. When electrons are emitted, the MITL current is calculated and compared with the magnetic insulation threshold. If the MITL current is higher than the magnetic insulation threshold, $J_{e}$ vanishes. The voltage vector derived in this step is the real value. If the MITL current is lower than the critical value, current loss exists. Then loss current density at that MITL element is calculated with Eqs. (3) to (7), and

$$
I_{\mathrm{loss}}(k, n)=J_{e} S,
$$

where $S$ is the equivalent cathode area of the MITL element.

At the same time, loss current is also the difference between two adjacent MITL elements. We have

$$
I_{\text {loss }}(k, n)=I(k, n)-I(k+1, n) .
$$

Then, with Eqs. (9) to (11), the current and the voltage vector at MITL element $k+1$ are updated in this step once more.

Strictly, the TL impedance $Z_{k}(n)$ changes with time, and should be updated at every time step in the magnetic insulation phase. The most commonly used model is the flow impedance model given by Mendel et al. [36]. Meanwhile, there is no suitable model to describe the physics of the phase between electron emission turn-on and the formation of magnetic insulation. On the other hand, updating impedance at every step may result in numerical instability or serious oscillation. Therefore, we use the vacuum impedance for this calculation. This kind of treatment is reasonable for the PTS, Z and ZR machine since in such high current facilities the duration between onset of electron emission and establishment of magnetic insulation is very short. Furthermore, the magnetic insulation in such systems is the so-called super insulation because of extremely high current. With these machines the electron sheath is very thin and close to the cathode surface in most areas. So the flow impedance of the MITL is very close to its vacuum value.

\section{SIMULATION RESULTS AND COMPARISON WITH EXPERIMENT}

As a numerical experiment, the code FAST is used to calculate the pulse transmission in the PTS for shot 0057, in
TABLE I. Marx charge voltage and load parameters for PTS shot 0057. The pinch resistance is assumed according to Refs. [11] and [33]. These parameters, together with the laser timing sequence, were used as input for the code FAST.

\begin{tabular}{lcccc}
\hline \hline $\begin{array}{l}\text { Marx } \\
\text { charging } \\
(\mathrm{kV})\end{array}$ & $\begin{array}{l}\text { Array } \\
\text { radius } \\
(\mathrm{mm})\end{array}$ & $\begin{array}{c}\text { Array } \\
\text { mass } \\
(\mathrm{mg} / \mathrm{cm})\end{array}$ & $\begin{array}{c}\text { Array } \\
\text { height } \\
(\mathrm{mm})\end{array}$ & $\begin{array}{c}\text { Pinch } \\
\text { resistance } \\
(\mathrm{m} \Omega / \mathrm{cm})\end{array}$ \\
\hline 65 & 26.4 & 2.3 & 15 & 5 \\
\hline \hline
\end{tabular}

which a tungsten array was fielded. The Marx charging voltage and load parameters for the shot 0057 are given in Table I. In order to run FAST, the timing sequence for all 12 triggering lasers should be specified. Water switches and prepulse switches were set to close when their voltage reaches the break down value derived in earlier experiments. Another set of parameters used to fit earlier experimental results are the coupling inductances between different MITL levels. The typical current fractions for level A to D MITLs in debugging experiments were 0.30, $0.26,0.24$ and 0.20 . To fit these fractions, a parametric sweep was done for determining coupling inductances between levels. (i.e., impedance for TL elements 196 to 198 as shown in Fig. 5 when transformed into the TL model). The values presently used are $0.2,0.8$, and $1.0 \mathrm{nH}$ between $\mathrm{A}$ and $\mathrm{B}, \mathrm{B}$ and $\mathrm{C}$, and $\mathrm{C}$ and $\mathrm{D}$ levels. In other words, the impedance for TL elements 196 to 198 are 10, 8, and $2 \Omega$. These values generally agree with the $3 \mathrm{D}$ static field calculation of DHPC inductance [37].

Typically, the calculation to $1500 \mathrm{~ns}$ from the beginning of the Marx discharge $(t=0)$ takes about 120 seconds for the code FAST with personal computers. This calculation time span covers the whole discharge and transmission process for an electromagnetic pulse in z-pinch experiments.

Figure 7 shows the load currents from the FAST calculation and the experiment measurements for shot 0057. The solid black line represents current recorded in the experiment and the red dashed line represents current from FAST with current loss. It can be seen that the consistency for the two curves is good, especially in the rising edge. The maximums are 8.8 and $8.75 \mathrm{MA}$ in the experiment and calculation, respectively. Figure 7 also shows with the green dot-dashed line the load current from FAST when no current-loss mechanism was introduced. This is what we could earlier get with a commercial circuit code. It can be inferred that if no mechanism is introduced to account for current loss during the formation of magnetic insulation, the calculated current curve will obviously diverge from the experimental curve in the rising edge. Because no loss exists after magnetic insulation establishes, which actually means that the loss and lossless models thereafter become the same, and the maximum currents for both calculations are almost identical. 


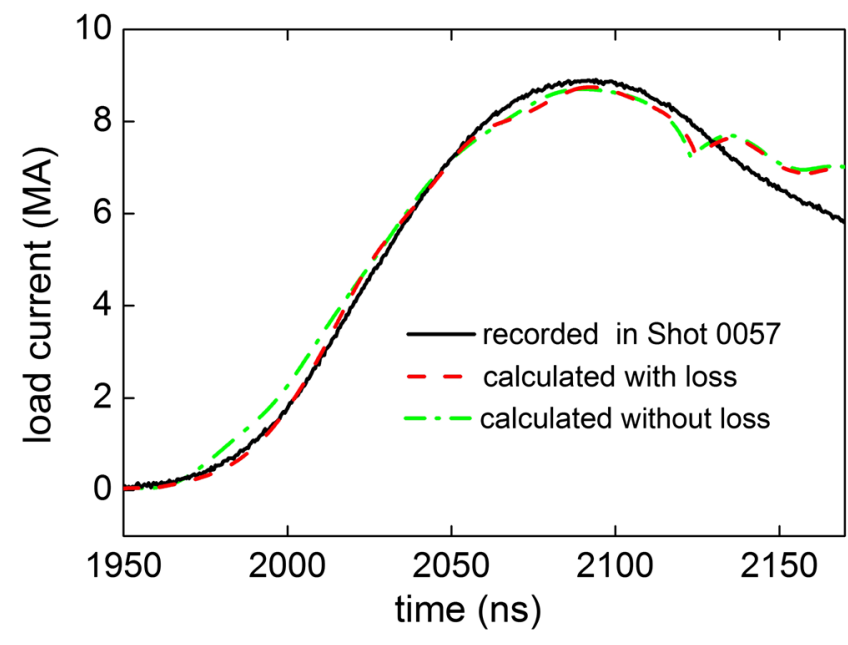

FIG. 7. Load current comparison of PTS shot 0057 and FAST calculations. The black solid line represents current recorded in the experiment, the red dashed line and the green dash dotted line represent calculated results with and without the loss mechanism. The consistency between the recorded result and the calculated result with loss mechanism suggests that the magnetic insulation criterion in the calculation is reasonable. The difference between the two calculated curves indicates that the current loss before magnetic insulation establishment occurs.

Current losses in each MITL level during the formation of magnetic insulation are shown in Fig. 8. Each curve represents the sum of current loss for all TL elements in that level. It indicates in Fig. 8 that current losses in the B and D levels are higher than in the A and $\mathrm{C}$ levels, and last much longer in time. This is mainly because for the $\mathrm{B}$ and $\mathrm{D}$ levels, higher critical currents are needed for magnetic insulation. As we know, the magnetic insulation critical current is higher with higher voltage and is inversely

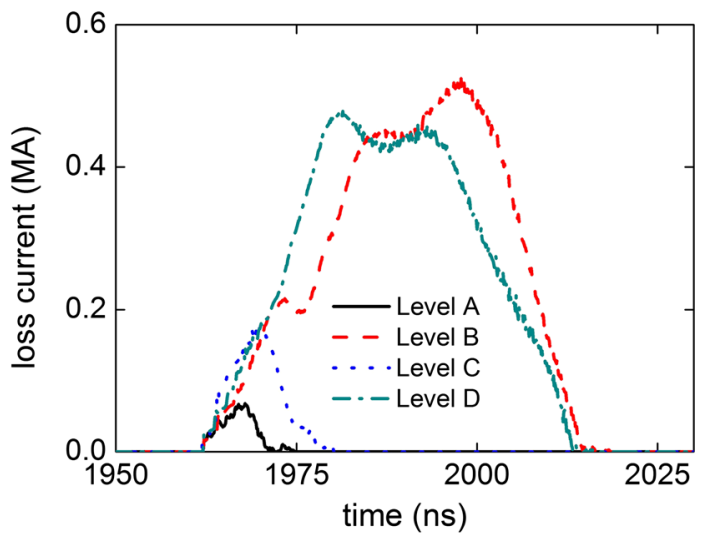

FIG. 8. Current loss in four MITL levels as a function of time. Current loss in the B (red dash) and D (dash dotted dark cyan) levels are higher than in the A (black solid) and C (blue dotted) levels, and last much longer in time. The difference in level voltage is the main reason for this, although impedance is another factor that takes current to a critical value.

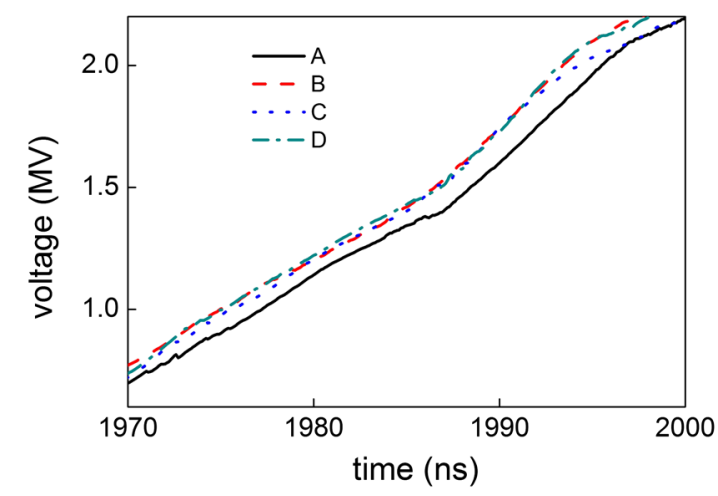

FIG. 9. Voltages of levels A to D between 1970 to 2000 ns. Voltage in level A (the black solid) is evidently lower than that in the others, and this leads to less loss. Voltage in level D (the dash dotted dark cyan) is slightly higher than that in level $\mathrm{C}$ (blue dotted) for most of the time since MITL elements in level D go into magnetic insulation later than those in level C. More current is lost in level $\mathrm{D}$.

proportional to vacuum impedance. As shown in Fig. 9, the voltage of level A is always lower than the others before 2000 ns. This makes level A go into magnetic insulation easier with lower current. The impedance of level A is lower too (Fig. 10), but here voltage becomes dominative with critical current. So less current loss exists in level A. This is the same case for levels C and D.

As indicated in Fig. 10, levels C and D have nearly identical impedance profiles along the transmission direction, although $\mathrm{D}$ has larger angles with respect to the horizon, and has a longer electrical length. But because of higher voltage, MITL elements in level D go into magnetic insulation later than those in level $\mathrm{C}$. Therefore, more current is lost.

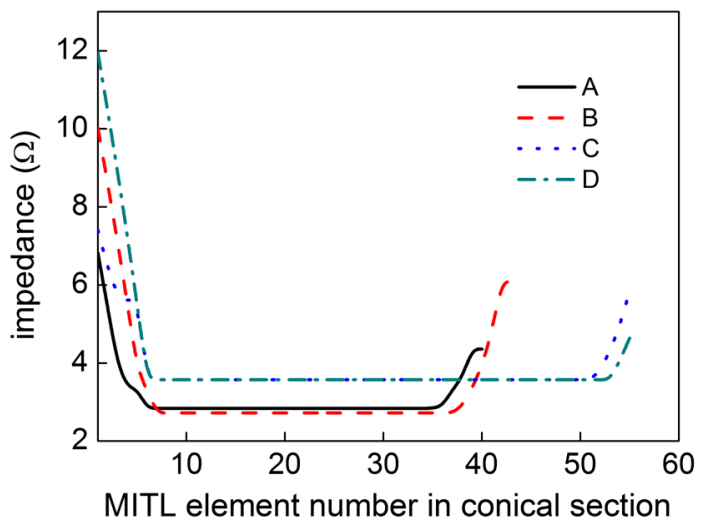

FIG. 10. Impedance profiles for level A to D in the transmission direction. The $x$ axis is the sequence number of MITL elements. All four levels have a section with uniform impedance. Levels A (the black solid) and $\mathrm{C}$ (the red dash) have lower impedance in general. Level C (blue dotted) and level D (dash dotted dark cyan) have longer electric lengths. 


\section{CONCLUSIONS AND DISCUSSIONS}

To conclude, a full circuit model for electromagnetic pulse transmission for the pulsed power driver PTS was introduced, in which all inductances and capacitances were transformed into TL elements, and the pulse transmission from the Marx generator to the load can be calculated with a TL algorithm. Time dependent characteristics were introduced into the corresponding code FAST. In particular, current loss and magnetic insulation in vacuum were taken into account. The code was tested with inputs from PTS shot 0057. Load currents from experiment and calculation show satisfactory agreement although presently crude models or parameters are used for some components. With the code run before the experiment, accelerator performance, working parameters for every component, power flow in MITL, energy loss in the pulse forming and transmission lines can be predicted with higher accuracy than before.

Although functional operation has been demonstrated, the circuit model and code need to be refined in the future. First, the resistance models for the laser switch, water switch and prepulse switches are identical and rough at present. The resistance changes from the opened value to the closed value within a specific transition time while, according to 3D electromagnetic computations by Rose et al. [17], those switches have more complex time-varying resistance histories. Second, the pinch resistance we used here is the same as in Ref. [11], although different array parameters were used in shot 0057. Frankly speaking, this is not reasonable. Calculating with a different pinch resistance, a different maximum load current would be obtained. Therefore, for quantitative prediction, a more dedicated pinch resistance model is preferred. Third, current loss in the inner MITL is ignored so far, so the load current equals the inner MITL current. In the future, a shunting mechanism should be incorporated to account for loss in the inner MITL. In experiments, the load current was measured with B-dots at the inner side of the anode post. This actually gives the total current flowing into the inner MITL. This is why only a slight difference exists between experimental and calculated current for shot 0057 at present.

\section{ACKNOWLEDGMENTS}

The authors would like to gratefully acknowledge Wei Bing, Ji Ce, Xia Minghe, Guan Yongchao, He Yong, Feng Shuping, and He An for fruitful discussion on the PTS circuit model, experimental settings and results. This investigation is partially supported by Science and Technology Development Grant of China Academy of Engineering Physics under Contract No. 2012A0402017.

\section{APPENDIX: DISCRETIZATION OF THE PTS TRANSMISSION NETWORK INTO TRANSMISSION LINE ELEMENTS}

To solve the telegrapher equation in a transmission line code, the whole transmission network is discretized into a series of short components which are then represented by short transmission line elements. The impedance $Z$ of the transmission line is related to the lumped inductance $L$ or the lumped capacitance $C$ of the component, by

$$
Z=\frac{L}{\tau}
$$

or

$$
Z=\frac{\tau}{C},
$$

where $\tau$ is the one-way transit time of the component. The determination of $\tau$ for each element mainly depends on the uniformity of the structure. For all lumped capacitance and inductance, $\tau$ is $0.1 \mathrm{~ns}$. For the components which change fast in structure, for example the water convolute, a smaller $\tau$ and more transmission line elements are desired.

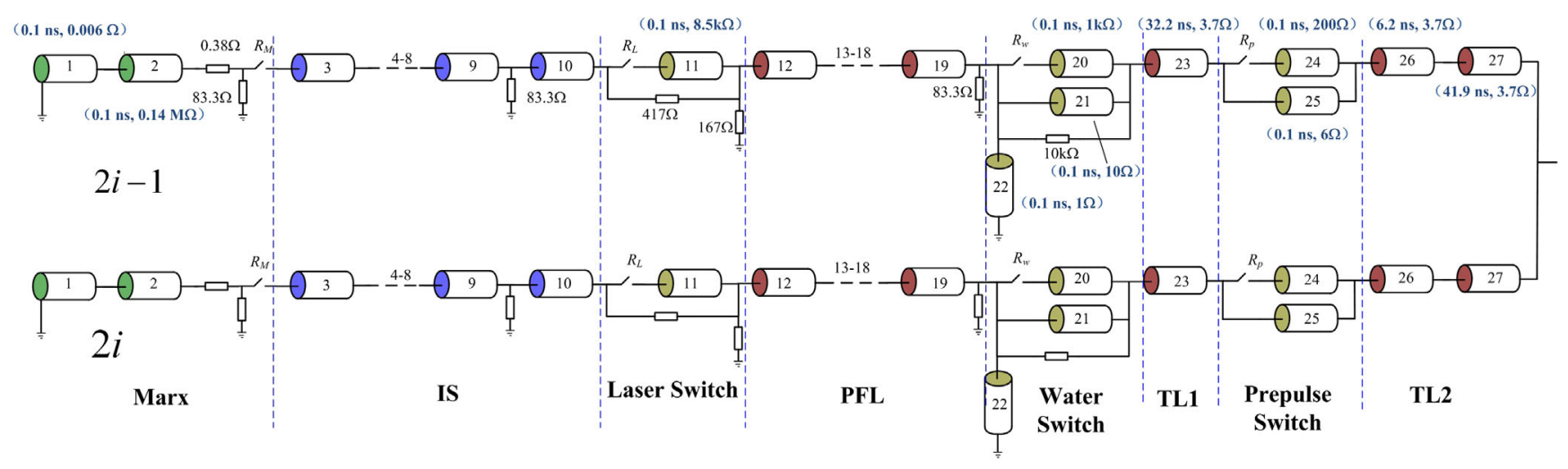

FIG. 11. Detail of the circuit before the water convolute. Module $2 i-1$ and $2 i$ are in the same angular position, sharing the same oil tank and water convolute. Before the water convolute, they have identical circuit parameters, which are shown in the figure. For all lumped capacitance and inductance, the transit length is $0.1 \mathrm{~ns}$, and the impedance is determined by Eqs. (A1) and (A2). The resistance for each component is fitted from experiments. 


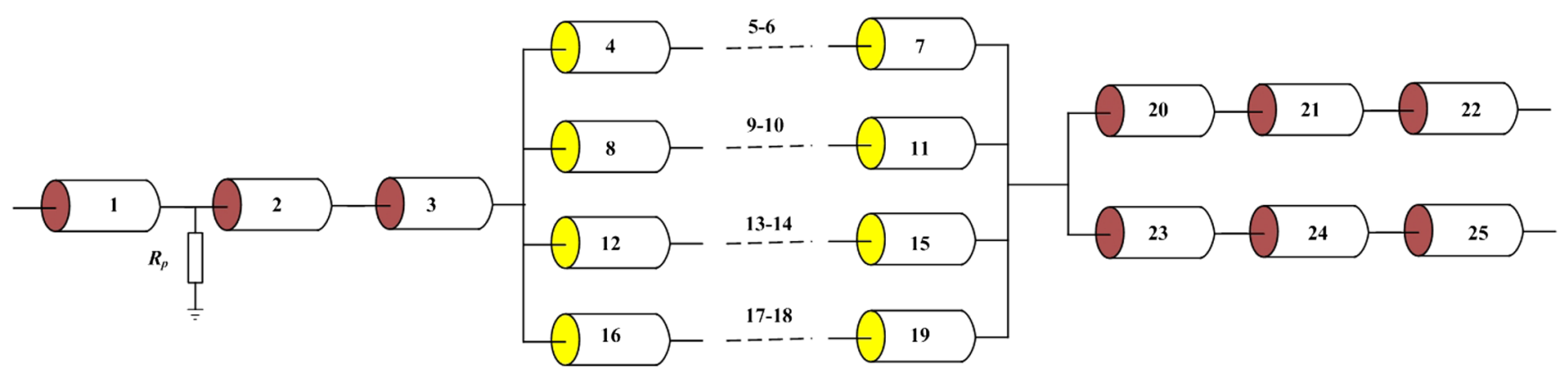

FIG. 12. Subcircuit of the water convolute in Fig. 3. The power from two adjacent modules merges after TL2 and is then transmitted downstream by a vertical triplate line (line 1 to 3 ). In the middle, the four levels represent the gradual transition from vertical to horizontal. When the transition is completed, two paralleled horizontal triplate lines connect to the insulation stack. Line 22 connects to levels $\mathrm{A}$ and $\mathrm{B}$, and line 25 connects to levels $\mathrm{C}$ and $\mathrm{D}$, of the insulation stack.

The representation of components by short line elements in effect denotes that the differential equations are approximated with finite difference equations and the operations of integration and differentiation are replaced by simple arithmetic operations.

In Fig. 3, for each module among the 24 ones, the Marx generator, the IS and the PFL each represents two, eight, and eight transmission line elements, respectively, and the TL1 represents one and TL2 represents two transmission line elements. All the above elements are in series. The detail of this part is shown by Fig. 11. Except for the IS and

TABLE II. Transit time in nanosecond and impedance in ohm for the transmission line elements in IS.

\begin{tabular}{lcc}
\hline \hline Element & Transit time $(\mathrm{ns})$ & Impedance $(\Omega)$ \\
\hline 1 & 0.8 & 62.0 \\
2 & 0.5 & 100.0 \\
3 & 11 & 6.5 \\
4 & 21 & 5.1 \\
5 & 21 & 5.1 \\
6 & 10 & 6.5 \\
7 & 0.5 & 63.0 \\
8 & 2.4 & 33.0 \\
\hline \hline
\end{tabular}

TABLE III. Transit time in nanosecond and impedance in ohm for the transmission line elements in PFL.

\begin{tabular}{lcc}
\hline \hline Element & Transit time $(\mathrm{ns})$ & Impedance $(\Omega)$ \\
\hline 1 & 1.3 & 31.9 \\
2 & 0.5 & 47.0 \\
3 & 6.4 & 5.0 \\
4 & 10 & 4.0 \\
5 & 9 & 4.0 \\
6 & 6 & 4.4 \\
7 & 0.5 & 37.0 \\
8 & 5.7 & 63.0 \\
\hline \hline
\end{tabular}

PFL, all other line parameters are illustrated in the bracket nearby. The resistance in each component is also indicated. The parameters for IS and PFL are shown by Tables II and III, respectively. The right side of Fig. 11 is connected to the left side of line 1 in Fig. 12, which represents the water convolute shared by two modules in the same angular position $(2 i-1$ and $2 i)$. The water convolute, which is divided into 25 line elements, gradually changes from one vertical triplate to two horizontal triplate lines. One of the horizontal triplate lines (20 to 22) connects to level

TABLE IV. Transit time in nanosecond and impedance in ohm for the transmission line elements in the water convolute.

\begin{tabular}{lcc}
\hline \hline Element & Transit time $(\mathrm{ns})$ & Impedance $(\Omega)$ \\
\hline 1 & 11 & 1.9 \\
2 & 1.8 & 2.1 \\
3 & 1.8 & 2.4 \\
4 & 1.8 & 11.1 \\
5 & 1.8 & 13.8 \\
6 & 1.8 & 15.5 \\
7 & 1.8 & 15.3 \\
8 & 1.8 & 10.0 \\
9 & 1.8 & 13.9 \\
10 & 1.8 & 15.6 \\
11 & 1.8 & 15.4 \\
12 & 1.8 & 10.0 \\
13 & 1.8 & 13.8 \\
14 & 1.8 & 15.5 \\
15 & 1.8 & 15.3 \\
16 & 1.8 & 11.1 \\
17 & 1.8 & 13.9 \\
18 & 1.8 & 15.6 \\
19 & 1.8 & 15.4 \\
20 & 1.8 & 7.5 \\
21 & 1.8 & 7.6 \\
22 & 1.8 & 6.7 \\
23 & 1.8 & 7.5 \\
24 & 1.8 & 7.6 \\
25 & 1.8 & 6.7 \\
\hline \hline
\end{tabular}


TABLE V. Transit time in nanosecond and impedance in ohm for the transmission line elements in the insulation stack.

\begin{tabular}{|c|c|c|c|c|c|c|c|c|}
\hline \multirow[b]{2}{*}{ Element } & \multicolumn{2}{|c|}{ Level A } & \multicolumn{2}{|c|}{ Level B } & \multicolumn{2}{|c|}{ Level C } & \multicolumn{2}{|c|}{ Level D } \\
\hline & $\begin{array}{c}\text { Transit time } \\
\text { (ns) }\end{array}$ & $\begin{array}{c}\text { Impedance } \\
(\Omega)\end{array}$ & $\begin{array}{c}\text { Transit time } \\
\text { (ns) }\end{array}$ & $\begin{array}{c}\text { Impedance } \\
(\Omega)\end{array}$ & $\begin{array}{c}\text { Transit time } \\
\text { (ns) }\end{array}$ & $\begin{array}{c}\text { Impedance } \\
(\Omega)\end{array}$ & $\begin{array}{c}\text { Transit time } \\
\text { (ns) }\end{array}$ & $\begin{array}{c}\text { Impedance } \\
(\Omega)\end{array}$ \\
\hline 1 & 1.8 & 1.1 & 1.8 & 1.1 & 1.8 & 1.1 & 1.8 & 1.1 \\
\hline 2 & 1.1 & 1.2 & 1.1 & 1.2 & 1.1 & 1.2 & 1.1 & 1.2 \\
\hline 3 & 0.8 & 1.2 & 0.8 & 1.2 & 0.8 & 1.2 & 0.8 & 1.2 \\
\hline 4 & 0.4 & 1.2 & 0.4 & 1.2 & 0.4 & 1.2 & 0.4 & 1.2 \\
\hline 5 & 0.3 & 1.4 & 0.3 & 1.4 & 0.3 & 1.4 & 0.3 & 1.4 \\
\hline 6 & 1.6 & 1.5 & 1.6 & 1.5 & 1.6 & 1.5 & 1.6 & 1.5 \\
\hline 7 & 0.6 & 1.6 & 0.6 & 1.6 & 0.6 & 1.6 & 0.6 & 1.6 \\
\hline 8 & 0.2 & 1.5 & 0.2 & 1.5 & 0.2 & 1.5 & 0.2 & 1.5 \\
\hline 9 & 0.3 & 8.4 & 0.3 & 8.4 & 0.3 & 8.4 & 0.3 & 8.4 \\
\hline 10 & 0.3 & 12.9 & 0.3 & 12.9 & 0.3 & 12.9 & 0.3 & 12.9 \\
\hline 11 & 0.1 & 16.8 & 0.1 & 16.8 & 0.1 & 16.8 & 0.1 & 16.8 \\
\hline 12 & 0.2 & 16.0 & 0.2 & 16.7 & 0.2 & 16.4 & 0.3 & 16.7 \\
\hline 13 & 0.1 & 15.2 & 1.2 & 11.3 & 0.4 & 14.3 & 0.1 & 18.1 \\
\hline 14 & 0.7 & 10.3 & 0.2 & 4.0 & 0.3 & 9.7 & 1.1 & 13.1 \\
\hline 15 & 0.2 & 4.1 & $\ldots$ & $\ldots$ & 0.2 & 6.6 & 0.2 & 5.9 \\
\hline
\end{tabular}

$\mathrm{A}$ and $\mathrm{B}$, and the other (line 23 to 25 ) connects to level $\mathrm{C}$ and $\mathrm{D}$ of the insulation stack. The parameters corresponding to Fig. 12 are shown by Table IV. Parameters for TL elements in the insulation stack are specified in Table $\mathrm{V}$

[1] R. B. Spielman et al., Phys. Plasmas 5, 2105 (1998).

[2] T. W. Sanford, R. E. Olson, R. L. Bowers, G. A. Chandler, M. S. Derzon, D. E. Hebron, R. J. Leeper, R. C. Mock, T. J. Nash, D. L. Peterson, L. E. Ruggles, W. W. Simpson, K. W. Struve, and R. A. Vesey, Phys. Rev. Lett. 83, 5511 (1999).

[3] M. K. Matzen, Phys. Plasmas 4, 1519 (1997).

[4] R. B. Spielman and J. S. De Groot, Laser Part. Beams 19, 509 (2001).

[5] D. D. Ryutov, M. S. Derzon, and M. K. Matzen, Rev. Mod. Phys. 72, 167 (2000).

[6] M. K. Matzen et al., Phys. Plasmas 12, 055503 (2005).

[7] D. J. Ampleford, C. A. Jennings, S. N. Bland, S. V. Lebedev, J. P. Chittenden, G. N. Hall, F. Suzuki-Vidal M. E. Cuneo, T. J. Rogers, M. Cleveland, R. D. McBride, J. D. Serrano, B. Peyton, C. A. Coverdale, M. C. Jones, and B. M. Jones, Report No. SAND2010-0833, 2010.

[8] E. V. Grabovskii, in Proceedings of the 5th International Conference on Dense Z-Pinches, Albuquerque, New Mexico, 2002.

[9] X. R. Kun, L. Z. Hong, N. J. Min, G. Cun, X. Z. Ping, Y. J. Lun, L. L. Bo, X. Xin, H. X. Sheng, D. Ning, L. Qiang, G. Y. Chao, E. V. Grabovsky, G. M. Oleynic, S. L. Nedoseev, and V. V. Alexandr, Chin. Phys. Lett. 22, 413 (2005).

[10] X. D. Ren, X. B. Huang, S. T. Zhou, S. Q. Zhang, J. Li, L. B. Yang, and P. Li, Acta Phys. Sin.. 58, 7067 (2009) (in Chinese).

[11] W. A. Stygar et al., Phys. Rev. ST Accel. Beams 12, 120401 (2009).

[12] R. B. Spielman, W. A. Stygar, J. F. Seamen, F. Long, H. Ives, R. Garcia, T. Wagoner, K. W. Struve, M. Mostrom,
I. Smith, P. Spence, and P. Corcoran, in Proceedings of the 11th IEEE International Pulsed Power Conference, edited by G. Cooperstein and I. Vitkovitsky (IEEE, Piscataway, NJ, 1997), p. 709.

[13] V. V. Aleksandrov, E. V. Grabovski, A. N. Gribov, G. M. Oleinik, A. A. Samokhin, and P. V. Sasorov, Plasma Phys. Rep. 34, 911 (2008).

[14] R. B. Spielman, in Proceedings of the 27th International Power Modulator Symposium, Washington, 2006 (IEEE, Piscataway, NJ, 2006), p. 43.

[15] K. W. Struve, T. H. Martin, R. B. Spielman, W. A. Stygar, P. A. Corcoran, and J. W. Douglas, in Proceedings of the 11th IEEE International Pulsed Power Conference (Ref. [12]), p. 162.

[16] LSP is a software product of ATK-Mission Research Corp. (http://www.mrcabq.com).

[17] D. V. Rose, D. R. Welch, E. A. Madrid, C. L. Miller, R. E. Clark, W. A. Stygar, M. E. Savage, G. A. Rochau, J. E. Bailey, T. J. Nash, M. E. Sceiford, K. W. Struve, P. A. Corcoran, and B. A. Whitney, Phys. Rev. ST Accel. Beams 13, 010402 (2010).

[18] R. Allen, P. Ottinger, and J. Schumer, in Proceedings of the 16th IEEE International Pulsed Power Conference, Albuquerque, NM (IEEE, Piscataway, NJ, 2007), p. 1095.

[19] P. A. Corcoran, in Proceedings of the 18th IEEE Pulsed Power Conference (IEEE, Piscataway, NJ, 2011), p. 103.

[20] D. D. Hinshelwood, NRL Memorandum Report No. 5185, 1983.

[21] W. N. Weseloh, in Proceedings of the 7th IEEE Pulsed Power Conference (IEEE, Piscataway, NJ, 1989), p. 990.

[22] S. Y. Song, Y. C. Guan, W. K. Zou, and J. J. Deng, in Proceedings of the 19th IEEE Pulsed Power and 40th Plasma Science Conference, San Francisco (IEEE, Piscataway, NJ, 2013).

[23] P. A. Corcoran, B. A. Whitney, V. L. Bailey, I. D. Smith, W. A. Stygar, M. E. Savage, G. A. Rochau, J. E. Bailey, B. M. Jones, T. J. Nash, M. E. Sceiford, L. G. Schlitt, and 
J. W. Douglas, in Proceedings of the 17th IEEE Pulsed Power Conference (IEEE, Piscataway, NJ, 2009), p. 150.

[24] P. A. Corcoran, J. W. Douglas, I. D. Smith, P. W. Spence, W. A. Stygar, K. W. Struve, T. H. Martin, R. B. Spielman, and H.C. Ives, in Proceedings of the 11th IEEE International Pulsed Power Conference (Ref. [12]), p. 466.

[25] D. Jianjun, X. Weiping, F. Shuping, W. Meng, L. Hongtao, S. Shengyi, X. Minghe, H. An, T. Qing, G. Yuanchao, G. Yongchao, W. Bin, Z. Wenkang, H. Xianbin, W. Yujuan, Z. Zhaohui, H. Yi, and Y. Libing, IEEE Trans. Plasma Sci. 41, 2580 (2013).

[26] F. Shuping, L. Hongtao, X. Weiping, D. Jianjun, X. Minghe, J. Ce, W. Meng, G. Yongchao, and H. An, High Power Laser Part. Beams 21, 463 (2009) (in Chinese).

[27] L. Hongtao, D. Jianjun, W. Yujuan, F. Shuping, X. Weiping, and D. Bonan, Plasma Sci. Technol. 10, 235 (2008).

[28] H. An, L. Fengping, D. Jianjun, L. Hongtao, F. Shuping, G. Yuanchao, and X. Weiping, in Proceedings of the First Euro-Asian Pulsed Power Conference, Chengdu, China, 2006, edited by D. Jianjun et al., Mianyang, China.
[29] A. A. Samokhin, Plasma Phys. Rep. 36, 682 (2010).

[30] C. A. JenningsJ. P. Chittenden, M. E. Cuneo, W. A. Stygar, D. J. Ampleford, E. M. Waisman, M. Jones, M. E. Savage K. R. LeChien, and T. C. Wagoner, IEEE Trans. Plasma Sci. 38, 529 (2010).

[31] J. Podsednik and D. Bliss, in Proceedings of the 14th IEEE Pulsed Power Conference, Texas, 2003, edited by M. Giesselmann and A. Neuber (IEEE, Piscataway, NJ, 2003), p. 1375.

[32] K. W. Struve, T. H. Martin, R. B. Spielman, W. A. Stygar, P. A. Corcoran, and J. W. Douglas, in Proceedings of the 11th IEEE International Pulsed Power Conference (Ref. [12]), p. 162.

[33] W. A. Stygar, G. A. Gerdin, and D. L. Fehl, Phys. Rev. E 66, 046417 (2002).

[34] A. A. Samokhin, Plasma Phys. Rep. 36, 149 (2010).

[35] A. Piel, Plasma Physics (Springer-Verlag Berlin, 2010), ISBN 978-3-642-10490-9, p. 170.

[36] C. W. Mendel, Jr. and S. E. Rosenthal, Phys. Plasmas 2, 1332 (1995).

[37] W. Qiang, W. Wendou, G. Yongchao, and S. Shengyi (private communication). 